\title{
Quantum-Inspired Feature and Parameter Optimisation of Evolving Spiking Neural Networks with a Case Study from Ecological Modeling
}

\author{
Stefan Schliebs, Michaël Defoin Platel, Sue Worner and Nikola Kasabov
}

\begin{abstract}
The paper introduces a framework and implementation of an integrated connectionist system, where the features and the parameters of an evolving spiking neural network are optimised together using a quantum representation of the features and a quantum inspired evolutionary algorithm for optimisation. The proposed model is applied on ecological data modeling problem demonstrating a significantly better classification accuracy than traditional neural network approaches and a more appropriate feature subset selected from a larger initial number of features. Results are compared to a Naïve Bayesian Classifier.
\end{abstract}

\section{INTRODUCTION}

$\mathbf{R}$ ECENTLY spiking neural networks (SNN) [1], [2] have been developed as biologically plausible connectionist models, which use trains of spikes for internal information representation. Today many applications using SNN receive a lot of research attention, some of them demonstrating very promising results on solving important real world problems. Based on [3] an evolving spiking neural network was proposed and applied to audio-visual pattern recognition [4], [5]. A similar type of network was later used in the context of a taste recognition task [6]. Other applications include e.g. neural based word recognition using liquid states [7], neural associative memory [8] and function approximation [9], just to name a few.

With encouraging results spiking neural networks were presented in the context of a feature selection problem [10]. In this work a state-of-art optimisation algorithm, namely the Versatile Quantum-inspired Evolutionary Algorithm (vQEA) [11], was combined with an Evolving Spiking Neural Network (eSNN) [4]. Implementing quantum principles vQEA evolves in parallel a number of independent probability vectors, which may interact at certain intervals with each other, forming a multi-model Estimation of Distribution Algorithm (EDAs) [12]. Following the wrapper approach, vQEA was used to identify relevant feature subsets and simultaneously evolve an optimal eSNN parameter setting. We will refer to this extended architecture as the Quantuminspired SNN (QiSNN) framework during the course of

Nikola Kasabov and Stefan Schliebs are with the Knowledge Engineering and Discovery Research Institute (KEDRI), Auckland University of Technology, New Zealand (e-mail: nkasabov@aut.ac.nz, sschlieb@aut.ac.nz; http://www.kedri.info).

Sue Worner is with the Lincoln University and Centre for Bioprotection, New Zealand (e-mail: worner@lincoln.ac.nz).

Michaël Defoin Platel is with the Biomathematics and Bioinformatics at Rothamsted Research, United Kingdom (e-mail: michael.defoinplatel@gmail.com) this paper. Applied to carefully designed benchmark data, containing irrelevant and redundant features of varying information quality, the QiSNN-based feature selection led to excellent classification results and an accurate detection of relevant information in the dataset.

This study intends to apply QiSNN on an ecological modeling problem. Meteorological data, such as monthly and seasonal temperature, rain fall and soil moisture recordings for different geographical sites, were compiled from published results. Furthermore for each global site the presence or absence of the Mediterranean fruit-fly (a serious fruit pest) was determined. Motivated by only inadequate results [13][15] using a different method, namely the Multi-layer Perceptron (MLP), this study aims towards the identification of important features relevant for predicting the presence/absence of this insect species. The obtained results may be also of importance to evaluate the risk of invasion of certain species into specific geographical regions.

In the following sections we will first present the QiSNN framework, explain the experimental setup along with a description of the data used, followed by an analysis and discussion of the obtained results.

\section{FRAMEWORK AND IMPLEMENTATION OF QISNN}

Based on our previous results on eSNN and quantum inspired evolutionary algorithms [3], [5], [12], [16], here we propose and explore an integrative quantum inspired feature selection using the eSNN architecture, tightly coupled with the learning environment (the data).

\section{A. eSNN Architecture}

The eSNN architecture uses a computationally very simple and efficient spiking neural model, in which early spikes, received by a neuron, are stronger weighted than later ones. The model was inspired by the neural processing of the human eye, which performs a very fast image processing. Experiments have shown that a primate only needs several hundreds of milliseconds to make reliable decisions about images that were presented in a test scenario [17]. Since it is known that neural image recognition involves several succeeding layers of neurons, these experiments suggested that only very few spikes could be involved in the neural chain of image processing. In [18] a mathematical definition of these neurons was attempted and tested on some face recognition tasks, reporting encouraging experimental results. The same 


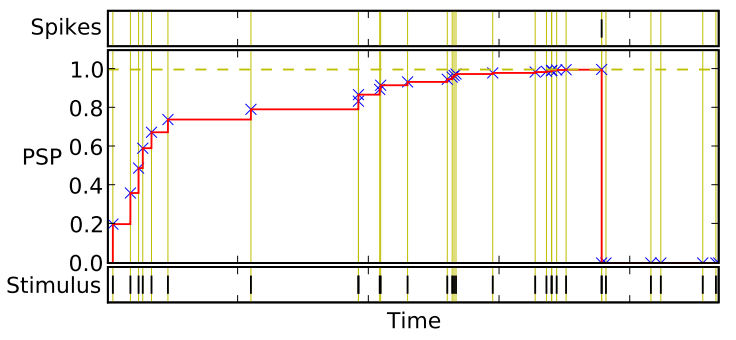

Fig. 1. Evolution of the PSP of a neural model used in QiSNN for a given input stimulus. If the potential reaches threshold $\theta$ a spike is triggered and the PSP set to 0 for the rest of the simulation, even if the neuron is still stimulated by incoming spike trains.

model was later used by [4], [19] to perform audio-visual face recognition.

Similar to other SNN approaches a specific neural model, a learning method, a network architecture and an encoding from real values into spike trains needs to be defined in the eSNN method. The neural model is given by the dynamics of the post-synaptic potential (PSP) of a neuron $i$ :

$$
\operatorname{PSP}_{i}(t)= \begin{cases}\sum_{j \mid f(j)<t} w_{j i} m_{i}^{\text {order }(j)} & \text { if fired } \\ \text { else }\end{cases}
$$

where $w_{j i}$ is the weight of a pre-synaptic neuron $j, f(j)$ the firing time of $j$, and $m_{i} \in(0,1)$ a parameter of the model, namely the modulation factor. Function $\operatorname{order}(j)$ represents the rank of the spike emitted by neuron $j$. For example a rank $\operatorname{order}(j)=0$ would be assigned, if neuron $j$ is the first among all pre-synaptic neurons that emits a spike. In a similar fashion the spikes of all pre-synaptic neurons are ranked and then used in the computation of $P S P_{i}$. A neuron $i$ fires a spike when its potential has reached a certain threshold $\theta$. After emitting a spike the potential is reset to $P S P_{i}=$ 0 . Each neuron is allowed to emit only a single spike at most. The threshold $\theta=c P S P_{\max }$ is set to a fraction $c \in(0,1)$ of the maximal potential $P S P_{\max }$ possible by a neuron. In Figure 1 the change of the PSP for this neural model is presented, when a series of input spikes (stimuli) are presented to the different synapses of this neuron.

An evolving neural network architecture using the above model along with a learning algorithm was proposed in [4], [5]. The method successively creates a repository of trained output neurons during the presentation of training samples. For each training sample a new neuron is trained and then compared to the ones already stored in the repository. If a trained neuron is considered to be too similar (in terms of its weight vector) to the ones in the repository (according to a specified similarity threshold $s$ ), the neuron will be merged with the most similar one. Otherwise the trained neuron is added to the repository as a new output neuron. The merging is implemented as the (running) average of the connection weights, and the (running) average of the two firing threshold. Because of the incremental evolution
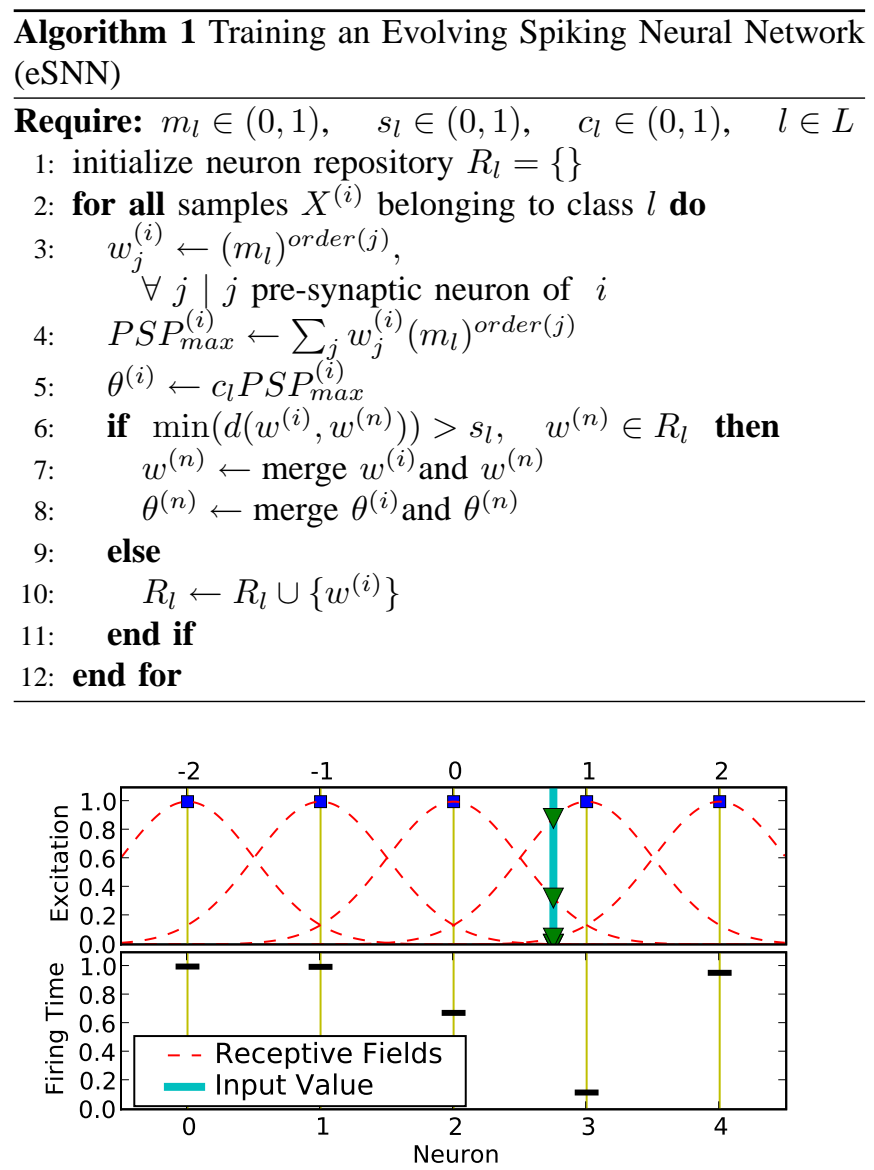

Fig. 2. Population encoding based on Gaussian receptive fields. For an input value $v=0.75$ (thick straight line in top figure) the intersection points with each Gaussian is computed (triangles), which are in turn translated into spike time delays (lower figure).

of output neurons it is possible to accumulate knowledge as it becomes available. Hence a trained network is able to learn new data without the need of re-training already learned samples. The procedure is described in detail in Algorithm 1.

Encoding of input values seems to be a critical factor in all SNN approaches. Several encoding mechanisms for SNN have been proposed, such as frequency mappings, Poisson processes and rank order encoding. Another approach is the population encoding which distributes a single input value to multiple neurons and hence may cause the excitation and firing of several responding neurons. Our implementation is based on arrays of receptive fields as described in [20], $c f$. Figure 2. Receptive fields allow the encoding of continuous values by using a collection of neurons with overlapping sensitivity profiles. Each input variable is encoded independently by a group of $M$ one dimensional receptive fields. For a variable $n$ an interval $\left[I_{\min }^{n}, I_{\max }^{n}\right]$ is defined. The Gaussian receptive field of neuron $i$ is given by its center $\left.\mu_{i}=I_{\text {min }}^{n}+(2 i-3) / 2 *\left(I_{\text {max }}^{n}-I_{\text {min }}^{n}\right) /(M-2)\right)$ and width $\sigma=1 / \beta\left(I_{\max }^{n}-I_{\text {min }}^{n}\right) /(M-2)$, with $1 \leq \beta \leq 2$. Parameter $\beta$ directly controls the width of each Gaussian receptive field. See Figure 2 for an example encoding of a single variable. Such eSNN architecture was applied to taste recognition 


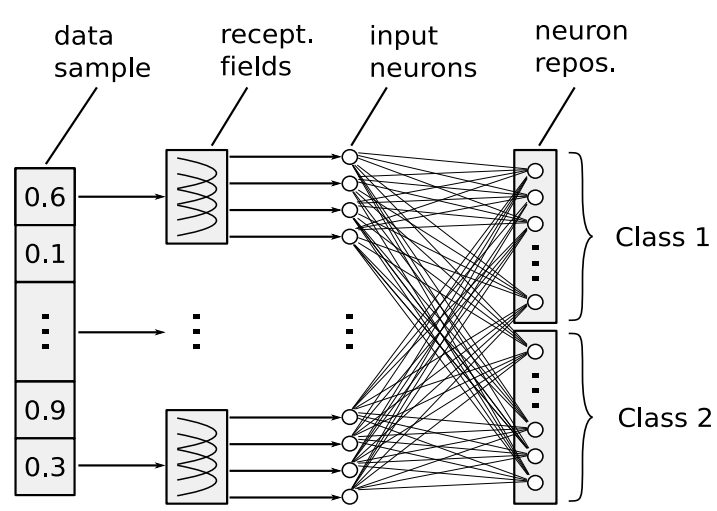

Fig. 3. eSNN architecture - Each input variable is translated into trains of spikes. The resulting spike sequence invokes a spiking neural network and a repository of output neurons is successively generated during the training process.

problem [6]. The described method is summarized in Figure 3 .

\section{B. Versatile Quantum-inspired Evolutionary Algorithm}

In this study eSNN was used to address feature subset selection (FSS) problems following the well known wrapper approach. A wrapper contains a general optimisation algorithm interacting with an induction method (classifier), also $c f$. Figure 5. The optimisation task consists in a proper identification of an optimal feature subset, which maximizes the classification accuracy determined by the inductor. The eSNN architecture operates as the induction method in this paper. Due to its interesting properties in terms of solution quality and convergence speed we decided for the previously proposed Versatile Quantum-inspired Evolutionary Algorithm (vQEA) [11] as the optimisation algorithm. The method evolves in parallel a number of independent probability vectors, which interact at certain intervals with each other, forming a multi-model Estimation of Distribution Algorithm (EDA) [12]. The use of a multiple probabilistic model is the main characteristic of vQEA, allowing a dynamical adaptation of the learning speed, which leads to a smooth convergence behavior. Furthermore it was shown that the multi-model works as a buffer against a finite number of decision errors. In previous work vQEA has been compared to Genetic Algorithms and a number of first level EDAs on several benchmark problems [11]. It has been shown that this approach performs well on epistatic problems, is very robust to noise, and needs only minimal fine-tuning of its parameters. In fact the standard setting for vQEA is suitable for a large range of different problem sizes and classes. Finally vQEA is a binary optimiser and fits well to the feature selection problem we want to apply it on.

\section{Integrated Parameter Optimisation}

Manual fine-tuning the neuronal parameters can quickly become a challenging task [19]. To solve this problem the idea of the simultaneous optimisation of the two combinatorial search problems of FSS and learning of parameters for the induction algorithm was proposed [21]. The selection of

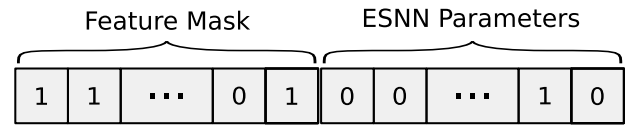

Fig. 4. Chromosome used in vQEA for simultaneously optimising feature and parameter space.

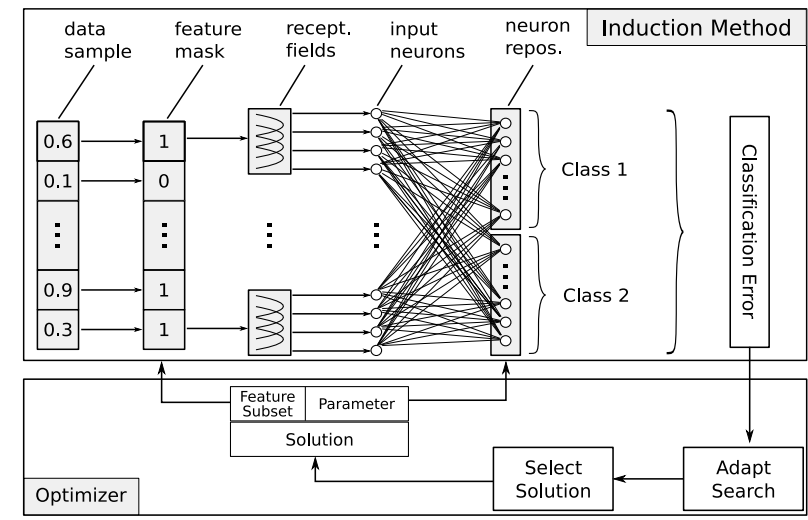

Fig. 5. The QiSNN framework of tightly coupled feature selection and parameter optimisation of eSNN, integrated with the data using vQEA.

the fitness function was identified to be a crucial step for the successful application of such an embedded approach. In the early phase of the optimisation the parameter configurations are selected randomly. As a result it is very likely that a setting is selected for which the classifier is unable to respond to any input presented, which corresponds to flat areas in the fitness landscape. Hence a configuration that will allow the network to fire (even if not correctly) represents a huge (local) attractor in the search space, which could be difficult to escape in later iterations of the search. In [21] a linear combination of several sub-criteria was used to avoid a too rugged fitness landscape. Nevertheless we can not confirm, that the use of much simpler fitness functions led to any problems in our experiments. Using the classification accuracy on testing samples seemed to work well as it is presented later in this paper. All parameters modulation factor $m_{l}$, similarity threshold $s_{l}$, PSP fraction $c_{l}, \forall l \in L$ of eSNN were included in the search space of vQEA. Due to its binary nature $v Q E A$ requires the conversion of bit strings into real values. As we will see later in the experimental analysis a small number of Grey-coded bits is sufficient to approximate meaningful parameter configurations of the eSNN method. In Figure 4 the structure of a chromosome as it is used in vQEA applied on eSNN is depicted.

The complete QiSNN framework used in this study is summarized in Figure 5.

\section{A CASE Study from Ecological Modeling}

Here we illustrate the inherent suitability of the QiSNN framework from Figure 5 to obtain better solutions of ecological problems characterised by large and changing data sets, large number of variables, and inadequate results obtained to date [13]-[15]. 


\section{A. Data}

For many invertebrate species little is known about their response to environmental variables over large spatial scales. That knowledge is important so that locations where a species that has potential to cause great environmental harm and might establish a new damaging population can be predicted. The usual approach to determine the importance of a range of environmental variables that explain the global distribution of a species is to train or fit a model to its known distribution using environmental parameters measured in areas where the species is present and where it is absent. In this study, meteorological data that comprised 68 monthly and seasonal temperature, rainfall and soil moisture variables for 206 global geographic sites were compiled from published records. These variables were correlated to global locations where the Mediterranean fruit-fly (Ceratitis capitata), a serious invasive species and fruit pest, were recorded at the time of the study, as either present or absent [22]. The dataset is balanced having equal number of samples for each of the two classes. Previous use of MLP on the data results in a classification accuracy of approximately $71 \%$ [14].

\section{B. Experiments}

In the proposed framework a number of parameter choices have to be made. For vQEA we chose a population structure of ten individuals organized in a single group, which is globally synchronized every generation. This setting was reported to be generally superior for a number of different benchmark problems [12]. The learning rate was set to $\theta=\pi / 200$ and the algorithm was allowed to evolve over a total number of 4000 generations. In order to guarantee statistical relevance 30 independent runs were performed, using a different random seed for each of them.

Additional to the feature space, vQEA was used to optimise the parameter space of the eSNN architecture. For each class $l \in L$ three parameters exist: The modulation factor $m_{l}$, the similarity threshold $s_{l}$, and the proportion factor $c_{l}$. Since the data represents a two-class problem, six parameters are involved in the eSNN structure. The binary character of vQEA requires the conversion of bit strings into real values. In the experiments we found four bits per variable enough to offer sufficient flexibility for the parameter space. For the conversion itself a Grey code was used.

In terms of the population encoding for eSNN we found that especially the number of receptive fields needs careful consideration, since it affects the resolution for distinguishing between different input variables. After some preliminary experiments we decided for 10 receptive fields, the centers uniformly distributed over the interval $[0,1]$, and the variance controlling parameter $\beta=1.5$.

In every generation the 206 samples of the dataset were randomly shuffled and divided into a training and testing set, according to a ratio of $75 \%$ (154 training and 52 testing samples). The chromosome of each individual in the population was translated into the corresponding parameter and feature space, resulting in the generation of a fully parameterized, but untrained eSNN and a feature subset. The created eSNN of each individual was then independently trained and tested on the appropriate data subsets. For the computation of the classification error we determined the ratio between correctly classified samples and the total number of testing samples.

In order to allow a comparison of the results we have also applied a traditional classification method on the same dataset by exchanging the eSNN classifier for the classical Naïve Bayesian Classifier (NBC). Using NBC in the wrapper approach is very common and was explored by many authors previously, e.g. [23]. Apart from the discretization of the dataset, which is a requirement for $\mathrm{NBC}$, all parameters as the training ratio and classification error metric were kept unchanged. For vQEA the learning rate was adapted to be $\theta=\pi / 100$, which we found to be in favour for the overall performance of the feature selection using NBC and the convergence speed.

\section{Results}

In Figure $6 a$ and $6 \mathrm{~b}$ the evolution through vQEA of the average best feature subset in every generation is presented using eSNN and NBC respectively as classifiers. The color of a point in this diagram reflects how often a specific feature was selected at a certain generation. The lighter the color the more often the corresponding feature was selected at the given generation. It can clearly be seen that a large number of features have been discarded during the evolutionary process. Many features have been identified to be irrelevant by both algorithms, although also significant differences between the evolved feature subsets can be noticed.

The eSNN classifier appears to be rather consistent in discarding features, since most of the 30 independent runs have agreed at least about the irrelevant features, hence many black columns appear in the diagram. The situation is different for features, that have been identified as relevant in most of the runs. In a small number of runs exactly these features were considered to be irrelevant, as reflected by the light gray columns in Figure 6a. For these features several hypothesis can be derived. We emphasize that the features for which the classifiers are undecided may be not important, but also not misleading during the evolutionary search. Hence they are randomly included in the final feature subset by any of the runs performed. It is also likely that some features are equally relevant (redundant features), so at least one of them will be selected as a representative of these features by the algorithm. Different runs will most likely select a different feature, thus the final subset is varying. We also believe that some features are present conditional to the presence/absence of others. Hence the average evolved feature subset can not be consistent in all runs and the ecological analysis of the feature subset should include all features that have been selected more frequent than a certain percentage in all runs performed.

In case of NBC a rather opposite situation can be observed. Some features are clearly found to be relevant in all 30 runs, which is in contrast to the results obtained by eSNN. But for many other features no definite decision can be 


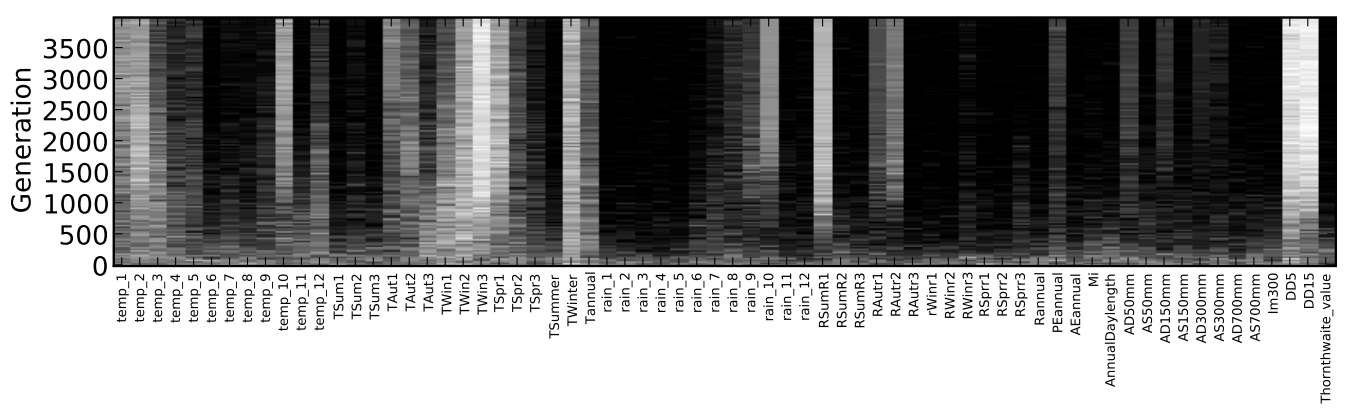

Feature

(a) Evolution of Generational Best Feature Subset using eSNN and vQEA

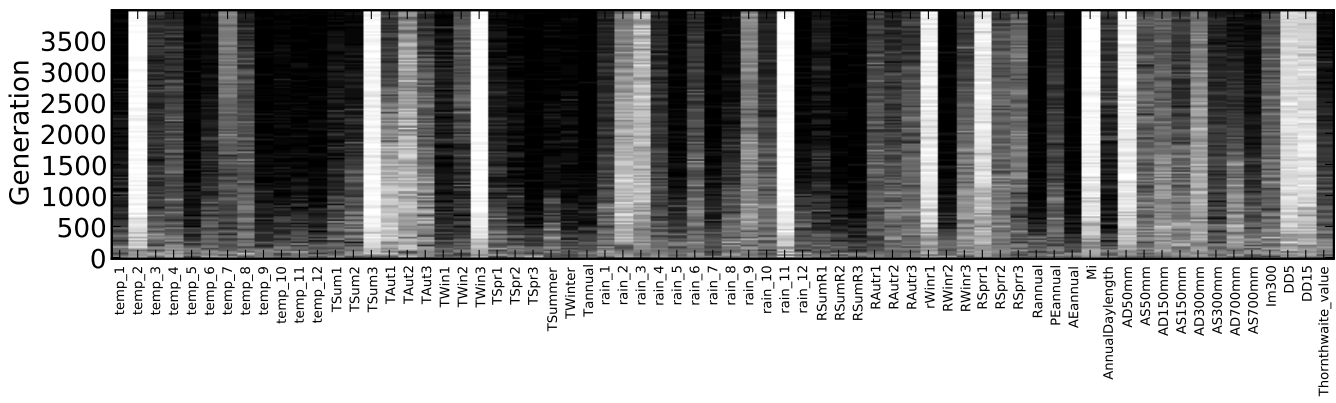

Feature

(b) Evolution of Generational Best Feature Subset using NBC and vQEA
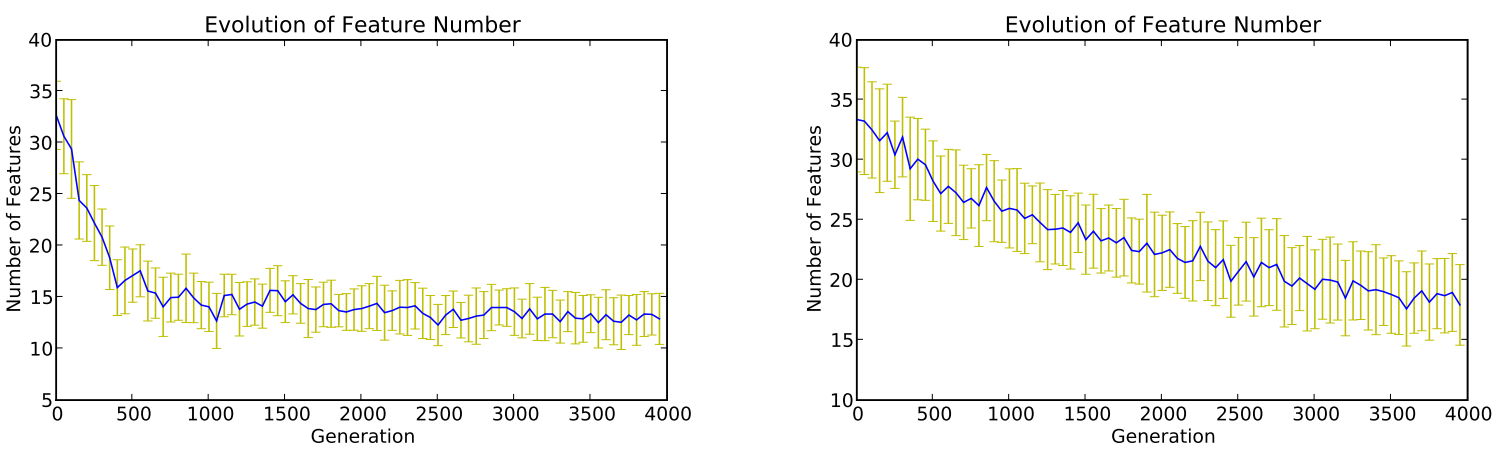

(c) Comparison of number of selected features between eSNN (left) and NBC (right), using vQEA
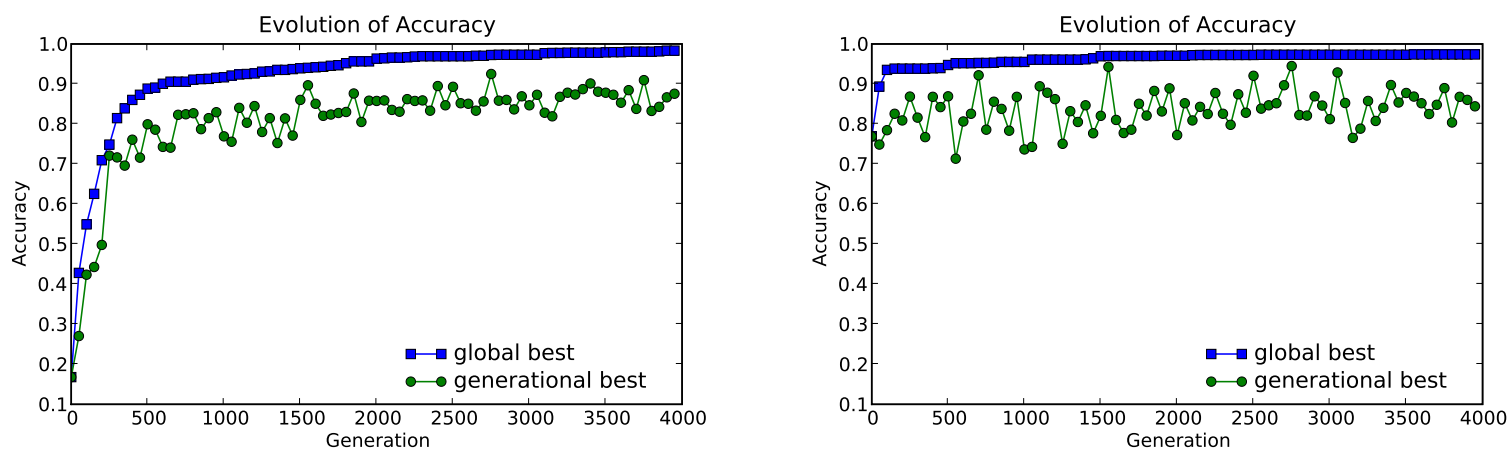

(d) Comparison of classification accuracy between eSNN (left) and NBC (right), using vQEA

Fig. 6. Results on the ecological data set averaged over 30 runs using different random seeds for the optimisation algorithm. (a), (b) The lighter the color of a point in the diagram, the more often a specific feature was selected at the given generation. Each point is the average of 30 independent runs. Many of these runs have identified similar relevant feature subsets, as indicated by a large number of either very dark or very bright points, but only very few gray ones. (c) In both algorithms the number of features decreases with increasing generations, eSNN being noticeably faster than NBC. (d) On the same time the eSNN classifier delivers a good estimate of the quality of the presented feature subset, while the trend of improved accuracy is less obvious using NBC. Continuing the evolutionary process beyond 4000 generations suggests further improvements in the classification accuracy, but also a decrease of the feature number. 
made, since some of the runs reported a given feature to be relevant, but on the same time an almost equal number of runs reported the exact opposite (reflected by the gray columns in Figure 6b). The explanations given earlier about redundant and conditional features are true for NBC, too.

The average number of features selected decreases steadily in later generations, but the trend in Figure $6 \mathrm{c}$ suggests the evolution for both algorithms is not completely finished, yet. After 4000 generations on average 14 features have been identified to be relevant using eSNN, 18 in the case of NBC. We will analyze these features from an ecological point of view in the next section.

The average accuracy reported by the best individual in the population after the evolution of 4000 generations, was constantly above $80 \%$ for both tested classifiers, NBC displaying a slightly higher variance during the evolutionary run compared to eSNN. It has to be noted that there is a difference about the way NBC and eSNN classify a test sample: While NBC always reports an answer (either class 0 or class 1 ), eSNN is also able to deny classification (either class 0 , class 1 or undecided). The latter case is considered to be a miss-classification of the presented sample. Hence the classification accuracy of NBC is at a very high level at the beginning of the evolutionary run, since a random classification would already correspond to an accuracy of approx. 50\%. While eSNN denies to report an answer for most presented test samples at the early stage of the optimisation, its classification accuracy starts at a much lower level than NBC. In later generations the accuracies of both algorithms can be compared fairly. Once more the overall trend suggests that the evolutionary optimisation could have been continued, expecting further improvement of classification accuracy. This is especially obvious for the eSNN based feature selection, but also in the case of NBC a small positive trend is noticeable.

We were interested how strong the classification accuracy is dependent on the feature number for each of the tested algorithms. In Figure 7 this dependence is investigated for both classifiers. Each point in the diagram corresponds to a tuple (accuracy, feature number) obtained from the generational best individual of every generation. The color indicates the generation itself, the lighter the color the later the generation in which a given tuple was obtained. In the case of eSNN ( $c f$. Figure 7a) a strong relationship between feature number and accuracy can be observed. Even for small decreases of the feature number significant accuracy improvements are reported. Since the evolutionary search is driven by the classification accuracy only, solutions having a small number of features represent a strong attractor in the search space.

In the case of NBC ( $c f$. Figure 7b) smaller feature subsets are also rewarded by higher classification accuracy. Nevertheless this award is less obvious compared to the one observed in eSNN. Thus the fitness landscape (in terms of feature number) represented by NBC appears to be more flat than the one represented by eSNN. It is noteworthy that flat fitness landscapes are an undesired property of any fitness function
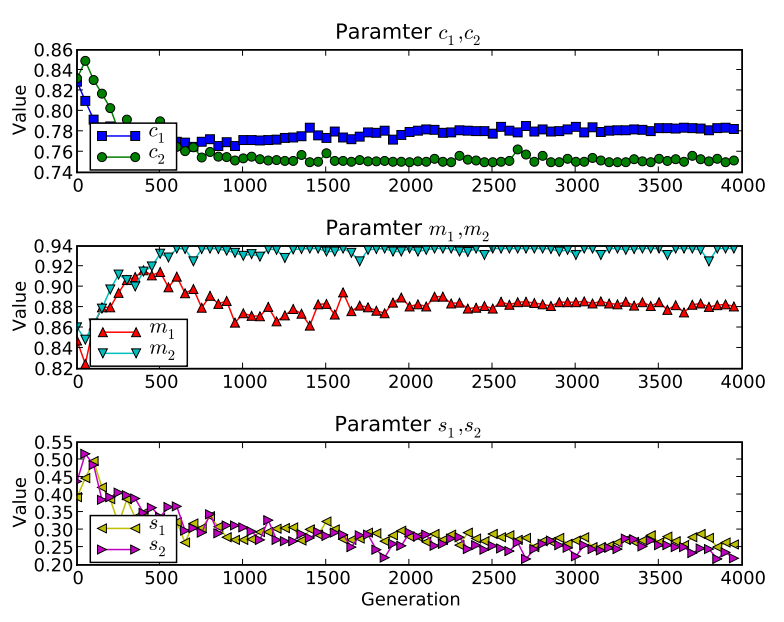

Fig. 8. Along with the features, the parameters of the eSNN model are optimised. Each parameter displays a rather smooth evolution, indicating the presence of careful control.

in an evolutionary algorithm.

Figure 8 presents the evolution of the parameters of the eSNN architecture. All three pairs display a steady trend and evolve constantly towards a certain optimum, not reporting too much variability. We take this as an indicator that vQEA indeed controlled these parameters carefully.

\section{Ecological Point of View}

Using the eSNN classifier on average only 14 features were selected in a particular evolutionary run, but since the evolved feature subsets were not identical in all of the runs and the presence/absence of features is also to be expected conditional to the presence/absence of other features, we have decided to include all features into the ecological analysis, that have been selected by at least $20 \%$ of the 30 independent runs. Thus in the case of eSNN the analysis indicates 25 variables that were considered as being involved in the determination of the classification outcome after the evolution of 4000 generations.

Table I summarizes the final feature subsets obtained by each of the classification methods. A feature is marked as rejected when at least $20 \%$ of all performed runs have discarded this feature at the end of the evolutionary run. If a feature was selected in $80 \%$ or more of all runs, it is marked as selected. Otherwise features have been labeled as "undecided" in the table. As mentioned earlier the table reflects the fact that eSNN is more consistent in rejecting features than NBC. For this reason we have concentrated our ecological analysis on the results of eSNN only. The features included in this analysis are presented by the two columns ("Undecided" and "Select") corresponding to the eSNN method in Table I.

Winter (TWIN2, TWIN3, TWINTER) and early spring (TSPR1) temperatures, and early summer rainfall (RSUMR1) were particularly strong features along with the degree-days (DD5 and DD15). Degree-days are the accumulated number 


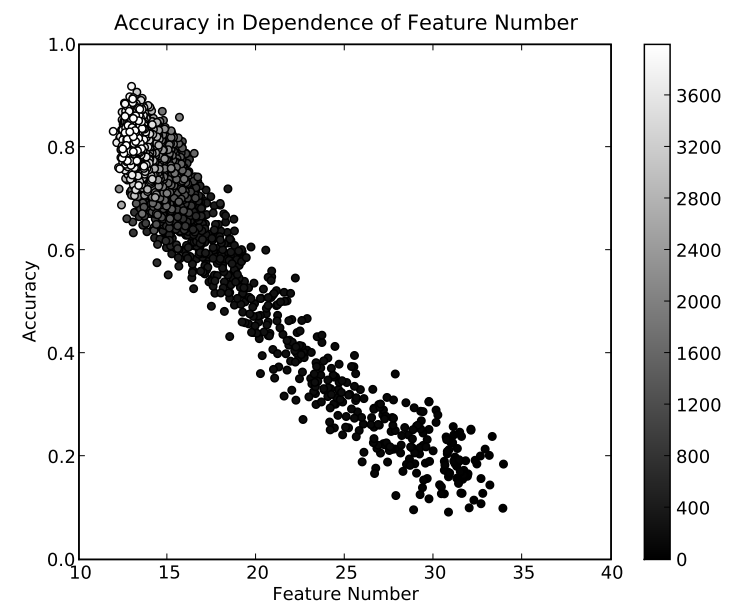

(a) eSNN

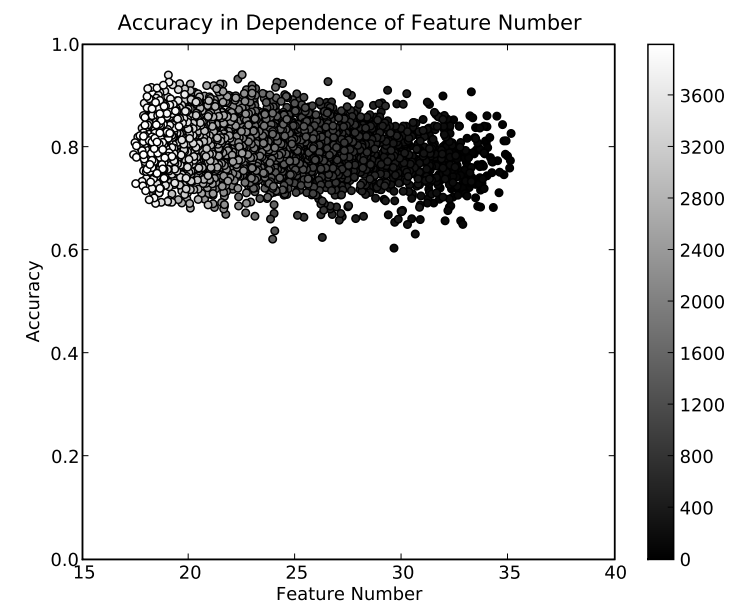

(b) NBC

Fig. 7. The diagrams show the accuracy as a function of the feature number for eSNN and Naïve Bayesian classifier. The different gray levels correspond to the generation in which a given data point was obtained. The lighter the color the later the generation. For eSNN the accuracy is highly dependent on the feature number, which is in strong contrast to NBC.

of degrees of temperature above a threshold temperature $\left(5^{\circ}\right.$ and $15^{\circ}$ in this case) over time (in this dataset over the whole year). It would be expected that the latter two variables would be closely correlated. These results correspond to other analysis where more conventional statistical and machine learning methods were used to identify the contribution of environmental variables to $C$. capitata presence or absence [24]. While there is no indication from this analysis whether the features have a negative or positive effect on the distribution of the species, it is known that $C$. capitata is limited by the severity of temperatures in the winter and early spring and extremes of wet or dry conditions in the summer [25].

The accuracy of the resulting model on the test set, however, is not only higher than that for the model using the full feature set, but also higher than that found by [24] using a range of conventional models. The clear potential for further improvement of classification accuracy with model refinement, as well as automatic optimisation of parameters, makes this an extremely useful approach for the analysis and modelling of complex, noisy ecological data.

\section{Conclusion And Future Directions}

In this study we have presented an extension of eSNN with a quantum-inspired evolutionary algorithm, which simultaneously evolves an optimal feature subset along with an optimal parameter configuration for eSNN. We call this framework QiSNN. We have used the already tested and published Versatile Quantum-inspired Evolutionary Algorithm. The framework was applied on an ecological data modeling problem and results have been compared to the traditional Naïve Bayesian Classifier. Although no significant difference in terms of accuracy between the two classification methods was obtained, some important experimental observations were made. While NBC represented a rather flat fitness landscape in the evolutionary algorithm in which lower numbers of features receive only little fitness rewards, eSNN reported a clear correlation between classification accuracy and feature number. As a result eSNN was capable of decreasing the feature number not only faster than NBC, but was also more consistent in excluding features from the optimisation process. NBC on the other hand appeared to be more consistent in selecting features, while being less consistent in rejecting them. The obtained feature subsets were analyzed by an ecological expert and found to be coherent with current knowledge in this area. In a previous analysis in which conventional statistical methods were applied on this dataset without performing any feature selection beforehand, a worse classification accuracy was reported.

Further development of the QiSNN framework is planned, where the presence/absence of spikes at a given time in a QiSNN will also be represented probabilistically with the use of quantum-inspired representation as suggested in [26], [27]. Also the rather strong disagreement of the investigated algorithms will be part of the future work.

\section{REFERENCES}

[1] W. Gerstner and W. M. Kistler, Spiking Neuron Models: Single Neurons, Populations, Plasticity. Cambridge, MA: Cambridge University Press, 2002. [Online]. Available: http://www.eur.nl/fgg/neuro/homepages/W.M.Kistler.htm

[2] E. M. Izhikevich, "Simple model of spiking neurons," IEEE Trans. on Neural Networks, vol. 14, no. 6, pp. 1569-1572, 2003.

[3] N. Kasabov, Evolving Connectionist Systems: The Knowledge Engineering Approach, 2nd ed. Secaucus, NJ, USA: Springer-Verlag New York, Inc., 2007.

[4] S. G. Wysoski, L. Benuskova, and N. Kasabov, "On-line learning with structural adaptation in a network of spiking neurons for visual pattern recognition," in $\operatorname{ICANN}(1), 2006$, pp. 61-70.

[5] _ - "Fast and adaptive network of spiking neurons for multi-view visual pattern recognition," Neurocomputing, vol. 71, no. 13-15, pp. $2563-2575,2008$.

[6] S. Soltic, S. Wysoski, and N. Kasabov, "Evolving spiking neural networks for taste recognition," in IEEE World Congress on Computational Intelligence (WCCI), Hong Kong, 2008. 


\begin{tabular}{|c|c|c|c|c|c|c|c|}
\hline Feature & Reject & $\begin{array}{c}\text { eSNN } \\
\text { Undecided }\end{array}$ & Select & Reject & $\begin{array}{c}\text { NBC } \\
\text { Undecided }\end{array}$ & Select & Agreement \\
\hline Temp1 & & yes & & yes & & & \\
\hline Temp2 & & yes & & & & yes & \\
\hline Теmp3 & & yes & & & yes & & $\mathrm{U}$ \\
\hline Temp4 & yes & & & & yes & & \\
\hline Temp5 & & yes & & yes & & & \\
\hline Temp6 & yes & & & yes & & & $\mathrm{R}$ \\
\hline Temp7 & yes & & & & yes & & \\
\hline Temp8 & yes & & & yes & & & $\mathrm{R}$ \\
\hline Temp9 & yes & & & yes & & & $\mathrm{R}$ \\
\hline Temp10 & & yes & & yes & & & \\
\hline Temp11 & yes & & & yes & & & $\mathrm{R}$ \\
\hline Temp12 & yes & & & yes & & & $\mathrm{R}$ \\
\hline TSum1 & yes & & & yes & & & $\mathrm{R}$ \\
\hline TSum2 & yes & & & yes & & & $\mathrm{R}$ \\
\hline TSum3 & yes & & & & & yes & \\
\hline TAut1 & & yes & & & yes & & \\
\hline TAut2 & & yes & & & yes & & U \\
\hline TAut3 & yes & & & yes & & & $\mathrm{R}$ \\
\hline TWin1 & & yes & & yes & & & \\
\hline TWin2 & & yes & & & yes & & U \\
\hline TWin3 & & & yes & & & yes & $\mathrm{s}$ \\
\hline TSpr1 & & yes & & yes & & & \\
\hline TSpr2 & & yes & & yes & & & \\
\hline TSpr3 & yes & & & yes & & & $\mathrm{R}$ \\
\hline TSummer & yes & & & yes & & & $\mathrm{R}$ \\
\hline TWinter & & yes & & yes & & & \\
\hline Tannual & & yes & & yes & & & \\
\hline Rain1 & yes & & & yes & & & $\mathrm{R}$ \\
\hline Rain2 & yes & & & & yes & & \\
\hline Rain3 & yes & & & & yes & & \\
\hline Rain4 & yes & & & & yes & & \\
\hline Rain5 & yes & & & yes & & & $\mathrm{R}$ \\
\hline Rain6 & yes & & & yes & & & $\mathrm{R}$ \\
\hline Rain7 & yes & & & yes & & & $\mathrm{R}$ \\
\hline Rain8 & & yes & & yes & & & \\
\hline Rain9 & & yes & & & yes & & \\
\hline Rain10 & & yes & & yes & & & \\
\hline Rain11 & yes & & & & & yes & \\
\hline Rain12 & yes & & & yes & & & $\mathrm{R}$ \\
\hline RSumR1 & & yes & & yes & & & \\
\hline RSumR2 & yes & & & yes & & & $\mathrm{R}$ \\
\hline RSumR3 & yes & & & yes & & & $\mathrm{R}$ \\
\hline RAutr1 & & yes & & & yes & & $\mathrm{U}$ \\
\hline RAutr2 & & yes & & yes & & & \\
\hline RAutr3 & yes & & & & yes & & \\
\hline RWinr1 & yes & & & & & yes & \\
\hline RWinr2 & yes & & & yes & & & $\mathrm{R}$ \\
\hline RWinr3 & yes & & & yes & & & $\mathrm{R}$ \\
\hline RSprr1 & yes & & & & & yes & \\
\hline RSprr2 & yes & & & & yes & & \\
\hline RSprr3 & yes & & & & yes & & \\
\hline Rannual & yes & & & yes & & & $\mathrm{R}$ \\
\hline PEannual & & yes & & yes & & & \\
\hline AEannual & yes & & & yes & & & $\mathrm{R}$ \\
\hline & yes & & & & & yes & \\
\hline ADayLen & yes & & & yes & & & $\mathrm{R}$ \\
\hline AD50mm & & yes & & & & yes & \\
\hline AS50mm & yes & & & & yes & & \\
\hline AD150mm & & yes & & & yes & & \\
\hline AS $150 \mathrm{~mm}$ & yes & & & & yes & & \\
\hline $\mathrm{AD} 300 \mathrm{~mm}$ & yes & & & & yes & & \\
\hline AS $300 \mathrm{~mm}$ & yes & & & yes & & & $\mathrm{R}$ \\
\hline AD700mm & yes & & & yes & & & $\mathrm{R}$ \\
\hline AS700mm & yes & & & yes & & & $\mathrm{R}$ \\
\hline $\operatorname{Im} 300$ & yes & & & & yes & & \\
\hline DD5 & & & yes & & & yes & $\mathrm{S}$ \\
\hline DD15 & & & yes & & yes & & \\
\hline Thornthw & yes & & & yes & & & $\mathrm{R}$ \\
\hline Total (\%) & 63.2 & 32.3 & 4.4 & 57.4 & 29.4 & 13.2 & 477.0 \\
\hline
\end{tabular}

TABLE I

FINAL FEATURE SUBSETS OBTAINED BY BOTH CLASSIFICATION ALGORITHMS. U=UNDECIDED, R=REJECTED, S=SELECTED

[7] D. Verstraeten, B. Schrauwen, and D. Stroobandt, "Isolated word recognition using a liquid state machine," in ESANN, 2005, pp. 435440.

[8] A. Knoblauch, "Neural associative memory for brain modeling and information retrieval," Inf. Process. Lett., vol. 95, no. 6, pp. 537-544, 2005.
[9] N. Iannella and L. Kindermann, "Finding iterative roots with a spiking neural network," Information Processing Letters, vol. 95, no. 6, pp. 545-551, 2005, applications of Spiking Neural Networks.

[10] S. Schliebs, M. Defoin-Platel, and N. Kasabov, "Integrated feature and parameter optimization for an evolving spiking neural network," in IEEE International Conference on Neural Information Processing, 2009, in Print.

[11] M. Defoin Platel, S. Schliebs, and N. Kasabov, "A versatile quantuminspired evolutionary algorithm," in IEEE Congress on Evolutionary Computation, 2007. CEC'07, 2007, pp. 423-430.

[12] — " "Quantum-inspired evolutionary algorithm: A multimodel eda," Evolutionary Computation, IEEE Transactions on, 2009, in print.

[13] S. Worner, G. Lankin, S. Samarasinghe, and D. Teulon, "Improving prediction of aphid flights by temporal analysis of input data for an artificial neural network," New Zealand Plant Protection, vol. 55, pp. 312-316, 2002.

[14] M. Watts and S. Worner, "Using mlp to determine abiotic factors influencing the establishment of insect pest species," in International Joint Conference on Neural Networks (IJCNN '06). Vancouver, Canada: IEEE, 2006, pp. 1840-1845.

[15] N. Cocu, R. Harrington, M. Rounsevell, S. Worner, and M. Hulle, "Geographical location, climate and land use influences on the phenology and numbers of the aphid, myzus persicae, in europe," Journal of Biogeography, vol. 32, no. 4, pp. 615-632, 2005.

[16] L. Benuskova and N. Kasabov, Computational Neurogenetic Modelling. NY: Springer, 2007.

[17] R. VanRullen and S. J. Thorpe, "Is it a bird? is it a plan? ultrarapid visual categorisation of natural and artificial objects," Perception, vol. 30 , pp. $655-668,2001$.

[18] S. J. Thorpe, "How can the human visual system process a natural scene in under 150ms? experiments and neural network models," in ESANN, 1997.

[19] S. G. Wysoski, "Evolving spiking neural networks for adaptive audiovisual pattern recognition," Ph.D. dissertation, Auckland University of Technology, August 2008, http://hdl.handle.net/10292/390.

[20] S. M. Bohte, J. N. Kok, and J. A. L. Poutré, "Error-backpropagation in temporally encoded networks of spiking neurons," Neurocomputing, vol. 48, no. 1-4, pp. 17-37, 2002.

[21] M. Valko, N. C. Marques, and M. Castelani, "Evolutionary feature selection for spiking neural network pattern classifiers," in Proceedings of 2005 Portuguese Conference on Artificial Intelligence, B. et al., Ed. IEEE, 2005, pp. 24-32.

[22] "Cabi (2003), crop protection compendium, global module, 5th edition," Wallingford, UK, 2003.

[23] E. Cantú-Paz, "Feature subset selection by estimation of distribution algorithms," in GECCO '02: Proceedings of the Genetic and Evolutionary Computation Conference. San Francisco, CA, USA: Morgan Kaufmann Publishers Inc., 2002, pp. 303-310.

[24] S. Worner, G. Leday, and T. Ikeda, "Uncertainty analysis and ensemble selection of statistical and machine learning models that predict species distribution," in Ecological Informatics, Cancun, Mexico, 2008.

[25] M. T. Vera, R. Rodriguez, D. F. Segura, J. L. Cladera, and R. W. Sutherst, "Potential geographical distribution of the mediterranean fuit fly, ceratitis capitata (diptera:tephritidae) with emphasis on argentina and australia," Environmental entomology, vol. 31, no. 6, pp. 10091022, 2002.

[26] N. Kasabov, "Integrative connectionist learning systems inspired by nature: Current models, future trends and challenges," Natural Computing, no. Jan, 2008.

[27] connectionist systems approach (feature article)," IEEE Computational Intelligence Magazine, vol. 3, no. 3, pp. 23-37, 2008. 